\title{
Las “Comme-fessions” de san Agustín. Apuntes sobre el giro espiritual de la fenomenología en J.-L. Marion*
}

\author{
Javier Bassas-Vila**
}

\begin{abstract}
Resumen: Recuperando ciertas tesis que expusimos en textos anteriores respecto a la importancia y las funciones de las partículas "como" y "como si" en el pensamiento de Jean-Luc Marion, el presente artículo propone una lectura de la reciente obra Au lieu de soi. L'approche de Saint Augustin del mismo pensador francés. Analizando las apariciones de dichas partículas en esta nueva obra, y con la intención de retomar y renovar el debate en torno al "giro teológico de la fenomenología francesa" (en palabras de D. Janicaud), proponemos ciertas hipótesis que apuntan a la posibilidad de una nueva relación entre teología y fenomenología con vistas a una concepción "espiritual" de esta última.
\end{abstract}

Palabras clave: Teología, Janicaud, como, como si, giro espiritual.

Abstract: This article suggests the reading of Jean-Luc Marion's recent work Au lieu de soi. L'approche de Saint Augustin bearing in mind some theses presented in previous articles concerning the importance and the functions of the particles "comme" and "comme si" ("as/like" and "as if") in the French author's thinking. By analyzing these particles in the text and with the intention of taking up and renew the debate on, in D. Janicaud's words, "the theological turn in French phenomenology", the article explores the potential for a new relationship between theology and phenomenology that leads us to a "spiritual" conception of the latter.

Key words: Theology, Janicaud, like, as, as if, spiritual turn.

Résumé: Nous reprenons certaines thèses que nous avons déjà exposées dans des textes précédents concernant l'importance et la fonction des particules "comme" et "comme si" dans la pensée de Jean-Luc Marion. Dans cet article, nous proposons une lecture de l'œuvre bien récente de celui-ci, Au lieu de soi. L'approche de Saint Augustin, en analysant les occurrences desdites particules dans cet ouvrage afin d'apporter une nouvelle perspective sur le débat autour du "tournant théologique de la phénoménologie française" (pour reprendre les mots de $\mathrm{D}$. Janicaud). Cet article propose certaines hypothèses qui visent la possibilité d'un nouveau rapport entre théologie et phénoménologie en vue d'une conception "spirituelle" de cette dernière.

Mots-clés: Théologie, Janicaud, comme, comme si, tournant spirituel.

* Una versión completamente española de este título podría ser: “Las Como-fesiones de san Agustín. Apuntes sobre el giro espiritual de la fenomenología en J.-L. Marion”. Más adelante, se explicará la razón del neologismo contenido en este título.

* Doctor en filología francesa y filosofía. Profesor Asociado, Universidad de Barcelona, España.

Recibido: 2010 - 10 - 17 (javierbassasvila@yahoo.es).

Aprobado: 2010 - 11 - 20 
En su gran obra titulada $A u$ lieu de soi. L'approche de Saint Augustin', Jean-Luc Marion se consagra a una lectura de las Confesiones de san Agustín para explicar, libro tras libro, la trayectoria recorrida por el pensamiento del obispo de Hipona. La pretensión general de esta lectura de las Confesiones consiste en extraer el contenido del pensamiento agustiniano de cualquier posible interpretación metafísica, concentrándose para ello en la cuestión de "sí", del sí mismo (en francés "soi"): ¿hay una definición del "soi" del hombre? ¿Podemos acceder a nuestro más íntimo interior, a nuestro "sí mismo"? Y, en tal caso, ¿qué encontraríamos entonces "en (el) lugar de" nuestra más íntima mismidad $^{2}$ ? Estas preguntas son las que conducen a Marion a analizar una gran cantidad de textos agustinianos con gran rigor $y$, sobre todo - es el primer punto que hay que señalar: una cuestión de método - a la luz del dispositivo teórico construido en su obra magna de 1997, Siendo dado ${ }^{3}$.

En efecto, la lectura marioniana de las Confesiones de san Agustín retoma las nociones más importantes que se exponían en Siendo dado: siguiendo la lectura de $A u$ lieu de soi, encontramos efectivamente el ego o el adonado (cap. II), la verdad o el fenómeno saturado (cap. III), la relectura no-metafísica de la voluntad (cap. IV) y el acontecimiento (cap. V), por no citar más

1 J.-L. Marion, Au lieu de soi. L'approche de Saint Augustin, Paris, Presses Universitaires de France, 2008.

2 Señalamos aquí que, en francés, el título del libro juega con la locución adverbial "au lieu de soi", significando al mismo tiempo "en el lugar de sí" y "en lugar de sí". Como en otros títulos de los libros de J.-L. Marion $($ Dieu sans l'être = Dios sin el ser; Étant donné = Siendo dado), se trata de una estrategia lingüística para dar a ver, desde un buen principio, de qué se tratará: en este caso, el título nos indica que lo que está en el seno mismo del hombre (su "soi") es quizá otra cosa que está "en el lugar de sí" y, por tanto, "en lugar de sí". El título Au lieu de soi podría entonces traducirse por En (el) lugar de sí.

3 Marion, J.-L., Étant donné. Essai d'une phénoménologie de la donation, Paris, Presses Universitaires de France, 1997; versión española: Siendo dado. Ensayo para una fenomenología de la donación, prólogo a la edición española de J.-L. Marion, traducción, presentación y notas de J. Bassas Vila, Madrid, Síntesis, 2008. que las nociones generales y sin entrar, por el momento, en el detalle de las argumentaciones que confirman explícitamente este vínculo de dependencia teórica entre ambas obras. No vamos, empero, a analizar en detalle este gesto metódico para saber si se trata de una aplicación extrínseca (y, por tanto, ilegítima) sobre un texto teológico o si el dispositivo teórico de Siendo dado nos ofrece, de hecho, un método de lectura que se puede generalizar para la interpretación de otras obras, filosóficas o no. Tampoco determinaremos si existe un paralelismo perfecto de las nociones que se exponen en las dos obras o si hay modificaciones sustanciales de una a otra obra. Dejamos todas estas cuestiones precisas para análisis por venir. Sin embargo, lo que sí nos parece importante - como un primer acercamiento general, necesario para entender esta obra - es advertir la manera como Marion justifica su lectura de las Confesiones de san Agustín a partir del dispositivo teórico de Siendo dado. A este respecto, leemos: "En un mot, nous assumons l'hypothèse que saint Augustin ignore superbement la distinction entre philosophie et théologie, parce qu'il n'appartient pas (encore) à la métaphysique. [...] Il guiderait par avance et sans intention préconçue nos pas hésitants en ayant pensé avant ce après quoi nous tentons de passer - la métaphysique et éventuellement l'horizon de l'être. [...] nous essaierons d'employer les concepts opératoires de la phénoménologie de la donation, pour mesurer s'ils ne se permettraient pas une lecture appropriée, cohérente et correcte des textes augustiniens" ${ }^{\prime 4}$.

4 Marion, Au lieu de soi, ob. cit., pp. 27-28. Sería interesante examinar el uso que Marion hace aquí de la noción "concepts opératoires" a la luz de las reflexiones de E. Fink sobre ese mismo tipo de conceptos en, por ejemplo, "Los conceptos operativos en la fenomenología de Husserl", donde leemos: son "esquemas intelectuales que en circunstancia alguna acceden a una determinación objetiva, representaciones mentales [Denkvorstellungen] abstractas que favorecen la aproximación a los conceptos temáticos y cuyo horizonte permanece opaco para el propio autor de la doctrina. Se trata, en suma, de conceptos que normalmente son utilizados [verbraucht] y que a veces incluso son llevados al límite de sus posibilidades [durchgedacht], pero que no llegan a ser propia- 
Esta cita confirma y detalla, de entrada, el vínculo teórico entre las dos obras o, más precisamente, la tentativa de aplicación del dispositivo teórico de Siendo dado a la interpretación de $A u$ lieu de soi.

Frente a este gesto metodológico, nos limitaremos a lanzar la siguiente reflexión. Que el pensamiento de Agustín sea pre-metafísico (lo cual es precisamente lo que debe demostrarse) podría volver coherente la lectura de sus textos a partir de un dispositivo que ya no se encuentra bajo el pensamiento metafísi$\mathrm{co}$, a saber, la fenomenología de la donación. Como Marion lo afirma claramente, de lo que se trata entonces es de leer un pensamiento que está antes de algo (el pensamiento de san Agustín en relación con la metafísica) a partir de un pensamiento que está después de algo (la fenomenología de la donación en relación con la metafísica). Ahora bien, que el pensamiento de Agustín pueda ignorar "soberbiamente [superbement]" la distinción entre filosofía y teología -indistinción que se erige como "el" rasgo no-metafísico - no implica necesariamente que nosotros, que pensamos después de que esta distinción haya imperado durante más de quince siglos en la historia del pensamiento, debamos o podamos olvidar esa misma distinción. En cualquier caso, ello constituye un nuevo acercamiento a la relación entre fenomenología (o, más generalmente, filosofía) y teología en el pensamiento marioniano, pues Siendo dado y en Dios sin el ser habían establecido, al contrario, una neta distinción entre estas dos disciplinas, por caminos diferentes ${ }^{5}$. Por el momento, afirmemos simplemente que las consecuencias de este cambio de perspectiva sólo pueden entenderse - más que por un juego histórico: lo que

mente pensados, ya que raramente son tomados en consideración [bedacht]", E. Fink, "Operative Begriffe in Husserls Phänomenologie", Zeitschrift für philosophische Forschung 11 (1957), p. 325.

5 En Siendo dado, puede leerse a este respecto la reflexión de Marion en torno a la distinción entre la descripción fenomenológica de la "revelación" (en minúsculas) y la aceptación por la fe de la "Revelación" (en mayúsculas), es decir, la distinción entre la posibilidad fenoménica de la revelación y la efectividad histórica de la Revelación - véase Siendo dado, ob. cit., p. 381. También pueden consultarse las reflexiones al respecto en Jean-Luc Marion, Dieu sans l'être, Paris, Presses Universitaires de France, 1982; versión española: Dios sin el ser, traducción de D. Barreto González, J. Bassas Vila y C. E. Restrepo, revisión y postfacio de J. Bassas Vila, Castellón, Ellago, 2010, especialmente el capítulo II, titulado "La doble idolatría". está antes o después de la metafísica - a partir del "giro espiritual de la fenomenología", tal y como aquí lo esbozaremos. Ésta es nuestra primera hipótesis, que retomaremos en nuestra tercera conclusión.

***

Pero dejemos esta cuestión así esbozada y abordemos, ahora sí, el tema que aquí nos interesa principalmente. Cabe recordar de entrada que, respecto al pensamiento de Marion, ya escribimos un postfacio para la edición española de Dieu sans l'être, titulado "Estudio de fenomenología lingüística. La historia del 'como' en Jean-Luc Marion"6, en el que se presentan los puntos fundamentales bajo un ángulo fenomenolingüístico. La lectura del presente artículo presupone, como se verá, algunas reflexiones que allí presentamos y a las que remitimos para más detalle. En ese postfacio, analizábamos de hecho todas las apariciones importantes de la partícula "como" y "como si" (en francés, "comme" y "comme si") en los textos marionianos fundamentales. Y reagrupábamos las apariciones de esas partículas bajo tres temáticas: primero, el debate en torno a la alabanza y los enunciados predicativos; segundo, el cambio de un cuestionamiento ontológico por un cuestionamiento más allá del ser; $y$, finalmente, las posibilidades que existen para establecer una descripción adecuada del fenómeno saturado. Lo que puede sorprender, de entrada, es constatar hasta qué punto estas partículas ("como" y "como si") están presentes en toda la obra marioniana en momentos clave de la reflexión. En efecto, ahí ya constatábamos textualmente que, entre muchos otros pasajes, esas partículas aparecían bajo una u otra forma: i) en el acto de enunciación de la alabanza, en el que la autoridad no corresponde a una institución humana, sino a Aquél que puede garantizar la veracidad del acto de alabar; ii) en la expresión de nuestra eventual indiferencia de ser, que se precisaba en el tedio, la vanidad y la melancolía, las tres disposiciones afectivas que imitan y remiten a la indiferencia de ser propia de Dios - tal y como

6 Véase Marion, Dios sin el ser, ob. cit., postfacio, pp. 315-356. 
ésta puede ser identificada en la lectura de ciertos pasajes de las Sagradas Escrituras: Romanos 4, 17, así como 1 Corintios 1, 28 o Lucas 15, 12-32 ; iii) el "como (si)" se manifiesta también textualmente en las reflexiones de Marion sobre nuestros actos morales, en los que no somos libres para decidir moralmente porque llevamos "el resplandor anónimo" de una "gloria" a la que remitimos - para retomar las mismas palabras del pensador francés-, gloria que nos precede y que no constituye ningún fundamento racional; iv) el "como (si)" aparece igualmente en la descripción marioniana del amor humano para marcar lingüísticamente la trascendencia de nuestro amor más allá del instante presente $\mathrm{y}$, así pues, con vistas a la eternidad - porque de nuestro amor no puede haber testimonio posible en este mundo (ni tan siquiera un hijo), ya que sólo puede testimoniar de nuestro amor el Amante perfecto que ya siempre nos ha amado, a saber, el Dios del amor (1 Juan 4, 8).

En los análisis que siguen, retomando precisamente esas reflexiones, nuestro propósito consiste en completar esta "historia del como" en la obra de Marion a partir de una lectura de Au lieu de soi. La historia del "como" quedará así más completa, iluminará tanto más claramente la obra marioniana y nos permitirá abrir nuevas preguntas respecto a la fenomenología, así como también nos permitirá comprender la radicalidad y la radicalización que ésta última gran obra propone de la relación entre teología y fenomenología - digamos provisionalmente, avanzando nuestros análisis, "la radicalización del giro teológico de la fenomenología", es decir, un "giro espiritual"7. Y todo ello, de hecho, demostrando una vez más la importancia que reviste el estudio de la escritura en los textos fenomenológicos, es decir, la importancia de su praxis de escritura.

$* * *$

7 Aceptemos, provisionalmente, que existe en el pensamiento de Marion lo que Janicaud entiende por "giro teológico", es decir, la ruptura de la inmanencia del a priori correlacional de la conciencia, tal y como lo expone Husserl, en favor de una trascendencia, es decir, de lo que excede a la conciencia misma. La radicalización de esto consistirá entonces en devolver, como aquí se verá más adelante, cierta inmanencia a la fenomenología a través de un reflexión que utiliza y pasa, pero no permanece, por la teología.
No resulta fácil analizar la obra de Marion titulada Au lieu de soi. Al número imponente de páginas, hay que añadir también la dificultad del contenido (el pensamiento de san Agustín), la alternancia constante entre dos lenguas (latín y francés), así como entre dos disciplinas (teología y fenomenología). Y, además, su reciente publicación. A día de hoy, apenas existen interpretaciones, comentarios o críticas sobre esta obra, sobre la pertinencia, el rigor o las lagunas de sus análisis: hasta donde sabemos, sólo disponemos de una breve recensión de Jean Grondin y algunos artículos publicados en 2009 en la Revue de métaphysique et de morale .

En primer lugar, nuestro propósito consiste en identificar las apariciones más importantes del "como" en Au lieu de soi. Y para decirlo claramente desde un buen principio: en $\mathrm{Au}$ lieu de soi, la importancia del "como" es más fundamental que en cualquier otra obra marioniana, ya que las funciones de esta partícula se multiplican en esta obra, se desarrollan al ritmo mismo de las argumentaciones y toman una profundidad fundamental en la construcción teórica del conjunto del libro. En efecto, hemos contabilizado hasta trece argumentaciones que se apoyan teóricamente en y se articulan lingüísticamente mediante el "como" y el "como (si)", siempre de una manera explícita9. Sin embargo, de las trece apariciones que hemos identificado, aquí sólo vamos a analizar cuatro: la primera y la segunda están relacionadas directamente con la alabanza y asocian así El ídolo y la distancia, así como Dios sin el ser, con estos últimos análisis de 2009; la tercera aparición insiste en la importancia que posee el "como" en la lectura de las Confesiones y abre la puerta a ese "giro espiritual de la fenomenología" que hemos anunciado; la cuarta aparición de la partícula "como" servirá para describir la posición del autor de las Confesiones mismas, a saber, la posición de san Agustín.

8 La recensión de Jean Grondin sólo es accesible en internet: http:// www.laviedesidees.fr/Au-lieu-de-la-metaphysique.html. Para el número de la Revue de métaphysique et de morale: "Saint Augustin, penseur du soi. Discussions de l'interprétation de Jean-Luc Marion", No. 63 (2009/3).

9 Véanse, al menos, las pp. 33, 88, 262, 318, 322, 324, 325, 330, 338, 352, 365,392 y 400 . 
Pero avancemos progresivamente, puesto que debe determinarse de entrada el marco general del acercamiento marioniano en $\mathrm{Au} \mathrm{lieu} \mathrm{de}$ soi. Marion empieza analizando las Confesiones a partir del género al que pertenecen, es decir, la "confessio" y, más precisamente, la alabanza. A este respecto, la posición que presenta es decisiva en la medida en que viene a completar y complicar el debate en torno a la predicación, la oración y la alabanza que tuvo lugar entre Derrida y Marion mismo. En efecto, en Au lieu de soi, Marion introduce y subsume un tercer término que no estaba presente de manera explícita en dicho debate: a la oración y la alabanza, habría que añadir ahora la "confesión" y los problemas conexos que ésta introduce. Y ello porque, según leemos en $\mathrm{Au}$ lieu de soi, la alabanza debe entenderse directamente a partir de la confesión, como una subdivisión de ésta que contendría también la "confessio peccatorum". Marion escribe en este sentido: "Nous admettrons d'abord et d'emblée le principe que, pour saint Augustin, la confessio se dédouble en confession des péchés et confession de louange"; y esta distinción tiene una función importante en la configuración del texto agustiniano y en la interpretación que construye Marion, quien añade seguidamente: "La louange constitue donc le point de départ des Confessions, qui provient d'elles en sorte qu'elles puissent finir pour s'accomplir toutes"; y concluye afirmando finalmente: "La louange parle en effet d'une parole qui ne prédique rien de Dieu, mais le confesse sans fin, parce que, face à son infinité, elle ne lui remontre pas"10. Es conocida la posición de Marion respecto a la alabanza: ésta es un acto lingüístico que no predica en el sentido tradicional de "A es b" (siguiendo la interpretación post-aristotélica del ti kata tinos como "enunciado"). Así pues, en estos fragmentos, Marion no está sino insistiendo en el hecho de que la alabanza no es una estructura predicativa; y lo hace afirmando que ésa no "advierte [remontre]" a Dios, sino que "reenvía [renvoie]" a Él y, por tanto, "remonta [remonte]" hacia Él para "hacerle frente [lui faire ainsi encontre]". La

10 Para las tres últimas citas, véase Marion, Au lieu de soi, ob. cit., respectivamente las pp. 31, 34 y 36. alabanza sigue siendo un acto no-predicativo, que viene ahora descrito triplemente en su relación con Dios: no advierte a Dios, sino que a Él reenvía y hacia Él remonta para hacerle frente (tal podría ser la traducción de las afirmaciones de Marion, que despliegan de hecho un juego de aliteraciones difícilmente traducible: "elle ne remontre pas à Dieu, mais renvoie et remonte vers lui pour lui faire encontre".

Ahora bien, siguiendo las últimas citas, lo que resulta más importante no es subrayar las coincidencias, sino sacar a la luz las novedades que la última obra de Marion introduce respecto a las obras precedentes: la alabanza está subsumida explícitamente a un acto lingüístico más general, a saber, la "confessio". Nos preguntamos entonces: ¿de qué manera la comprensión y subsunción de la alabanza estricta y explícitamente a partir de la "confessio", y junto a la "confessio peccatorum", podría completar o complicar el debate entre Derrida y Marion? ¿Cómo podría retomarse este debate a partir de estos tres actos lingüísticos: la alabanza, la oración y la confesión en sus tres modalidades específicas (confesión de pecados, confesión de alabanza y confesión de fe)? ¿Qué relaciones se establecen entre ellos y, más precisamente, entre las tres modalidades de la confesión? ¿Son las tres igualmente no-predicativas? A pesar de existir análisis de la confesión de fe en, por ejemplo, Dios sin el ser ${ }^{11}$, parece que todo lo expuesto al respecto en $A u$ lieu de soi abre nuevos caminos aún por recorrer.

11 En Dios sin el ser pueden encontrarse efectivamente algunos análisis de la "confesión de fe" que prefiguraban, ya en 1982, las reflexiones al respecto en Au lieu de soi. La coherencia del pensamiento de Marion y la obsesión por unas mismas preguntas saltan entonces a la vista, tras más de 30 años de estudios minuciosos. Entre varias afirmaciones sobre la "confesión de fe" en la obra de 1982, retengamos las siguientes (el primer número de página corresponde a la edición francesa): "La difficulté de ramener à l'unité les différents termes du discours [le sujet et le prédicat, "Jesus" et "Seigneur"] semble moins affaiblir ou interdire la confession de foi que, d'abord, la caractériser en pro-

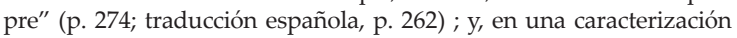
en términos positivos, Marion escribe: "La confession de foi passe par l'énonciateur, mais elle vient de plus loin et va plus loin" (p. 275; traducción española, p. 263), o "En renonçant à performer et à prédiquer selon un modèle de maîtrise, celui qui confesse 'Jésus [est] Seigneur' performe pourtant déjà un acte d'amour, prédique pourtant déjà correctement du Verbe qu'il peut aimer. Sans doute cette confession reste-t-elle inchoativement ordonnée à la charité. Mais cet écart lui-même n'échappe point à la charité. Car l'écart implique l'abandon, et surtout l'endurance de cet abandon. Aussi bien la confession se trouve-t-elle reprise par le martyre" (p. 276; traducción española, p. 263). 
Continuemos ahora reflexionando sobre la alabanza mediante un breve análisis de la primera y segunda aparición de la partícula "como". Marion escribe: "D'emblée, il est clair qu'aucun nom unique ne suffit à nommer Dieu; d'abord, évidemment parce qu'il s'agit de le louer comme... et qu'un tel comme... interdit une nomination univoque, mais déploie un écart, tel qu'il interdit de jamais prétendre saisir la moindre définition conceptuelle univoque"12. Esta cita no parece aportar nada nuevo respecto a lo que ya hemos concluido aquí y a lo que ya expusimos más detalladamente en el citado postfacio a la edición española Dios sin el ser: encontramos de entrada el marco general de la cuestión en la que se inscribe la alabanza, a saber, la problemática en torno a los nombres de Dios; también encontramos el efecto de distanciamiento ("écart") del "como", efecto que impide captar una definición unívoca de Dios - diríamos, más técnicamente, que imposibilita una identificación entre el contenido de la intención de significación de la alabanza y la intuición impletiva propia de la manifestación de Dios. En este mismo sentido, la segunda aparición del "como" en Au lieu de soi confirma esta misma caracterización de la alabanza. En efecto, a propósito de "idipsum" (el "nombre" de Dios que san Agustín propone, según la lectura de Marion, y que resulta no ser un nombre sino un deíctico $-y$ esto sí que constituye un nuevo rasgo en el pensamiento de Marion respecto a los nombres de Dios), podemos leer lo siguiente: "Par conséquent, attribuer une signification fixe à idipsum constitue une absurdité et une faute de logique, qui ravale la dénomination comme... au rang d'une nomination, à la fois impraticable et illégitime ${ }^{\prime \prime 13}$. Estas dos apariciones del "como" propio de la estructura lingüística de la alabanza sirven simplemente para confirmar la continuidad en la reflexión marioniana de un mismo marco general y una misma posición respecto a la alabanza, así como para aportar algunos nuevos matices en la descripción de este mismo acto lingüístico. Au lieu de soi se mantiene, en este sentido, en la senda trazada

12 Marion, Au lieu de soi, ob. cit., p. 392.

13 Ibídem, p. 400. por El ídolo y la distancia, así como por Dios sin el ser o el último capítulo de De surcroît, por no citar más que los textos más importantes sobre la alabanza y la predicación ${ }^{14}$.

Ahora bien, la tercera y cuarta aparición de la partícula "como" que queremos identificar y analizar en las argumentaciones de Au lieu de soi aportan nuevas e interesantes reflexiones. Éstas explicitan la manera como deben ser entendidas la lectura y la escritura de las Confesiones de san Agustín.

Resulta que ambas operaciones - la lectura y la escritura de las Confesiones - están fundamentalmente determinadas por el "como". Notemos de entrada lo que dice Marion sobre la lectura: "La première phrase des Confessions s'articule donc d'emblée en une demande (Dieu se donne à louer), puis en une réponse (de fait l'homme le loue, comme aussi toute création). Du même coup, s'esquisse la possibilité d'une difficulté herméneutique: si celui qui ne loue pas ne peut approcher Dieu et si les Confessions veulent approcher Dieu, alors tout lecteur qui se refuserait à louer s'interdirait du même coup d'entendre et même de lire les Confessions. Et alors, l'obstacle herméneutique découlerait d'un refus proprement spirituel". Marion está afirmando aquí que el acto de leer el texto de las Confesiones (si es que se puede hablar de texto, tomemos provisionalmente el término "texto" en sentido laxo) implica una actitud especial, un cambio de actitud, una disposición espiritual adecuada. Ahora bien, lo que nos interese poner de relieve es que ese cambio de actitud necesario para la lectura del texto agustiniano viene marcado textualmente por Marion mediante un "como". En efecto, Marion añade a este pasaje una nota fundamental que retoma la cuestión de lo "espiritual" explícitamente e introduce la cuestión del "como". En esta nota, leemos: "Autrement dit, le 'comme' herméneutique dépend d'un 'comme' plus originaire, qu'on ne qualifiera pourtant pas ici d'existential, ni de

14 Véase mi artículo crítico sobre el uso que Marion hace del "como" de la alabanza en estos mismos textos en "Écriture phénoménologique et théologique", publicado en C. Ciocan y M. Neamtu (eds.), Studia Phaenomenologica, 2009, volumen titulado Philosophical concepts and religious metaphors: new perspectives on phenomenology and theology. 
phénoménologique, mais de spirituel. À moins de supposer qu'en son fond la phénoménologie puisse devenir elle-même une affaire de spiritualité" 15 . Como puede comprenderse, esta nota es efectivamente fundamental y concentra en ella la posible radicalización del giro teológico de la fenomenología francesa del que hemos hablado y que aquí se enunciaría explícitamente, según nuestra interpretación, como un giro "espiritual" de la fenomenología: "À moins de supposer qu'en son fond la phénoménologie puisse devenir elle-même une affaire de spiritualité", escribe Marion.

En la breve recensión que Jean Grondin redactó sobre $A u$ lieu de soi, puede leerse lo siguiente: "Mais si l'ego se trouve secondarisé chez Marion, c'est parce qu'il se reçoit d'ailleurs que de soi, savoir d'un don primordial, qui ne peut être que divin. La phénoménologie devient ici une 'affaire de spiritualité', qui continuera d'en faire sourciller certains. Faut-il lui préférer une phénoménologie sans esprit? Si un esprit sans phénoménologie est aveugle, une phénoménologie sans esprit reste vide"16. La cuestión merece análisis más precisos que aquí tan sólo podemos esbozar y, de entrada, la comprensión de qué debe entenderse por "espiritual". Porque, ¿qué es lo que Marion quiere sugerirnos con esta enigmática, breve, pero fundamental nota? ¿Y cómo leer esta nota dentro del conjunto de su pensamiento? Intentemos dar un principio de respuesta.

De hecho, no se trata de constatar simplemente que el espíritu participa en la fenomenología de alguna manera, sino que se trata más bien de sugerir que lo propio de la fenomenología es una "cuestión de espiritualidad" y, por consiguiente, que el método fenomenológico debe ser pensado como una cuestión, no de razón, sino de actitud espiritual. Aquí nos avanzamos evidentemente por la senda sugerida por Marion, aunque no explicitada en esa misma obra. Ahora bien, a pesar de necesitar análisis más profundos, nos permitimos correr

15 Ibídem, respectivamente p. 33 y la nota 1 de esta misma página. 16 Véase la citada recensión de J. Grondin, referenciada más arriba. aquí cierto riesgo para apuntar la manera como debe entenderse lo "espiritual" en la reflexión marioniana: corremos, pues, el riesgo de postular que esa "cuestión de espiritualidad [une affaire de spiritualité]" a la que se hace referencia en la nota debe ser entendida en el pensamiento de Marion a partir de sus reflexiones en torno a la voluntad. $\mathrm{O}$, para decirlo de una manera más precisa: cuando Marion escribe que el "como" hermenéutico cede la primacía a un "como espiritual" en la lectura de las Confesiones de san Agustín y que ese "como espiritual" podría ser, "en su fondo", lo propiamente fenomenológico, lo que en realidad está haciendo es extremar las hipótesis que ya había anunciado en Siendo dado, a saber: que es la voluntad la que permite $\mathrm{y}$, por tanto, determina en última instancia la manifestación propia de los fenómenos. O para más brevemente: para ver, hay que querer ver, y ese "querer" abre una nueva figura no-metafisica de la voluntad - Le croire pour le voir, reza el título del último compendio de artículos de Marion ${ }^{17}$. Con ello, lo que estamos haciendo es situar esta "cuestión de espiritualidad" en el plano de las "decisiones extra ratione" que ya advertimos en nuestro postfacio a Dios sin el ser ${ }^{18}$, a saber, que la efectuación del acto moral de los Prolegómenos a la caridad, la reducción fenomenológica del don en Siendo dado y la descripción del amor de los amantes en $E l$ fenómeno erótico se efectúa mediante un acto de voluntad que fenomenaliza lo que, de otra manera, no podría volverse fenómeno. A nuestro entender, faltos de más tiempo para la reflexión (de ahí el carácter de "apuntes" de estas reflexiones), la relación entre fenomenología y "la cuestión de espiritualidad" que Marion sugiere en esa nota pasa entonces por la voluntad y sus posibles figuras no-metafísicas, así como, en consecuencia, por una redefinición de la verdad a partir de la manifestación del fenómeno saturado o, dicho en palabras de Au lieu de soi, por una definición de la verdad, ya no como "adecuación", sino como "veritas lucens" y "veritas redarguens". Volveremos sobre ello.

17 Véase J.-L., Marion, Le croire pour le voir, Paris, Parole et silence, 2010.

18 Véase Dios sin el ser, edición española citada, p. 340. 
De momento, notemos que los análisis del "como" adquieren así un mayor alcance, diríamos precisamente infinito. Resumamos brevemente los resultados conseguidos hasta ahora. Por el momento, hemos constatado que el "como" aparece en las reflexiones de Au lieu de soi en, al menos, dos puntos esenciales: primero, el "como" de la (confesión de) alabanza que abre y cierra las confesiones; segundo, el "como" de la lectura que puede abrir a una comprensión espiritual de la fenomenología. Ahora bien, resulta que a todo esto debe añadírsele una última aparición del "como", la cuarta aparición de esta partícula que queremos analizar de entre las trece que hemos identificado en las argumentaciones de $A u$ lieu de soi.

Como ya habíamos avanzado, también la escritura de las Confesiones debe concebirse mediante una operación articulada por el "como". A este respecto, Marion nos hace ver de entrada hasta qué punto la escritura de una confesión religiosa, sea cual sea, resulta una aporía: "Et si l'éternité [de Dieu] n'a rien de commun avec le temps, à proprement parler aucune confessio, pas plus de louange que d'aveu, n'a de sens, parce qu'elle n'a pas lieu de jamais se produire. En stricte philosophie, Dieu n'a pas à répondre à la moindre confessio, parce qu'il n'a pas à en connaître". La escritura de las Confesiones de san Agustín queda, por tanto, directamente afectada: "À bien le considérer, cette aporie a déjà silencieusement menacé, ou plutôt, en droit, c'est-à-dire philosophiquement, elle a disqualifié toute la confessio depuis le début de son déploiement théologique de fait. Saint Augustin a écrit jusqu'ici comme si la confessio pouvait déployer son entreprise, alors qu'en toute rigueur métaphysique elle ne le pouvait pas, puisque les dieux n'entendent pas les hommes, ni ne s'y entendent" ${ }^{\prime 19}$. Dos observaciones al respecto:

i) primero, siguiendo lo dicho en esta nota, el "como si" nos da de nuevo acceso a cierta relación con la eternidad, es decir, con un tiempo esencialmente otro que no es el tiempo humano y que resulta, para noso-

19 Marion, Au lieu de soi, ob. cit., p. 262. tros (ad nos), incomprensible. Por tanto, de igual manera que en El fenómeno erótico la resolución de los amantes intenta cumplirse para toda la eternidad y no lo consigue si no es mediante un "como si" que pretende convocar a Dios como testigo, así también constatamos ahora que la (confesión de) alabanza y la confesión de pecados no pueden llevarse a término si no es mediante un "como si" que intenta crear un acceso a la eternidad de Dios. Dado que Dios no tiene que conocer nuestros pecados o nuestras alabanzas en el tiempo, la redacción de las Confesiones padece entonces de una paradoja que no puede evitarse más que por un "como si". El "como si" asumen aquí el rol de un operador lingüístico que convierte en aceptable la eternidad de Dios, para nosotros mortales, sin pretender asimilarla;

ii) en segundo lugar, cabe señalar que el "como si" de la escritura de las Confesiones abre una interpretación original y rigurosa del célebre capítulo XI de esa misma obra. En efecto, la reflexión de san Agustín sobre el tiempo ya no debe entonces comprenderse a partir de las conocidas y tradicionales interrogaciones: según Marion mismo, el capítulo XI no es ni una reducción psicológica del tiempo, ni una anticipación de la doctrina kantiana ni una pre-comprensión existencial del ser, sino que " [...] il s'agit de concevoir comment le temps ne ferme pas plus à l'éternité qu'il ne s'y abolit bref comment il pourrait s'articuler sur elle sans confusion, ni séparation" ${ }^{20}$. Es decir, el capítulo XI de las Confesiones abre así una comprensión de la relación entre el tiempo (humano) y la eternidad, en la que ambas "temporalidades" no se pretenden fusionables ni están completamente separadas. Reencontramos aquí confirmada la función de "distanciación" del "como (si)", su acto de aproximación-sin-identificación, su "écart (distancia)" irreductible.

20 Ibídem, p. 263. 
En definitiva, la importancia del "como" en $A u$ lieu de soi no deja lugar a dudas, pues resulta fundamental:

i) en la alabanza y, más precisamente, en la comprensión de la "alabanza" como "confesión" con su efecto de distanciación irreductible ("écart");

ii) en la lectura de las Confesiones y la abertura a una comprensión "espiritual" de la fenomenología que apela a una nueva concepción de la verdad y que se relaciona con una nueva figura post-metafísica de la voluntad, con las decisiones extra rationes, así como con la veritas lucens y la veritas redarguens que describe Marion en esa misma obra - veritas lucens/redarguens que no son más, de hecho, que una concepción explicitada por la teología de la verdad asociada a la fenomenalización del fenómeno saturado;

iii) en la escritura misma de las Confesiones, evitando la aporía que consiste en escribir en el tiempo - alabar y confesar en el tiempo humano - ante la eternidad de Dios - que está fuera del tiempo.

Llegados a este punto, propongamos entonces cuatro conclusiones:

Primera conclusión: por todo lo dicho hasta aquí - aunque esquemática y brevemente-, nos sentimos autorizados a afirmar que las Confesiones de san Agustín devienen bajo la interpretación marioniana las "Comme-fessions" de san Agustín, es decir, una confesiones determinadas por el "como" (en francés, "comme"), unas Como-fesiones o, menos abruptamente en español, las Confesiones en como: tanto su contenido - la alabanza-, como su lectura y su escritura son inseparables del "como" y sus diversas funciones precisas.

Segunda conclusión: ahora bien, la interpretación marioniana de las Comme-fessions ha demostrado la posibilidad entreabierta de una radicalización del giro teológico de la fenomenología francesa. Tenemos, por una parte: i) las diversas argumentaciones preparadas en libros anteriores: las decisiones extra ratione y la figura post-metafísica de la voluntad, junto a la nueva concepción de la verdad en Au lieu de soi como "veritas lucens" y "veritas redarguens" (insistimos en que esta nueva concepción remite a la manifestación particular del "fenómeno saturado", la cual no es sino una cuestión de aceptación o rechazo, antes bien que una cuestión de "adecuación", es decir, que se trata de una llamada y no una adecuación ${ }^{21}$ ). Y tenemos, por otra parte: ii) esa sugerencia en nota de que la fenomenología, "en su fondo [en son fond]", sería una "cuestión de espiritualidad". Todo ello nos parece apuntar más allá de cualquier giro "teológico" y afirmar la necesidad de un nuevo examen de la relación entre una y otra disciplina, así como una posible nueva concepción de la fenomenología misma. Sin embargo, poner en relación "la espiritualidad" y la "fenomenología" es abrir una caja de truenos. De entrada, podemos preguntarnos al respecto: ¿debemos vincular esta espiritualidad estricta y únicamente a la teología? Y, ¿no podríamos entender una espiritualidad propiamente fenomenológica, al margen de la teología? Tal es, a nuestro entender, la posibilidad más interesante que nos ofrece actualmente el pensamiento marioniano: "la cuestión de espiritualidad" abierta por la relación entre teología y fenomenología acaba superando la teología misma para abrir la posibilidad de una "espiritualidad" propiamente fenomenológica.

Tercera conclusión: siguiendo las dos conclusiones precedentes y todo lo dicho aquí, podemos entender mejor de qué manera y por qué pueden aplicarse los "conceptos operativos" expuestos en la fenomenología de la donación a la lectura de una obra teológica como son las Confesiones de san Agustín. En efecto, respecto a la primera "cuestión de método" que hemos evocado, concluimos que esta aplicación interpretativa no se justifica tanto mediante una argumentación "histórica" (podemos pensar lo que está antes de la metafísica - el pensamiento de san Agustín - mediante lo que está después

21 Véase al respecto, por ejemplo, Siendo dado, ob. cit., pp. 420 y ss. 
de la metafísica - la fenomenología de la donación-), sino mediante una reflexión sobre las "afinidades" epocales: la fenomenología de la donación desemboca en un giro espiritual de la fenomenología que permite precisamente su acercamiento y su aplicación a la teología. Ahora bien, insistimos en que la puerta abierta que nos parece más fecunda en este sentido es la que nos conduce a pensar una espiritualidad propiamente fenomenológica, liberada de cualquier reducto teológico, especialmente, de la comprensión de lo espiritual a través de una trascendencia mediada por la fe. ¿No se trataría, más bien, de repensar la función de una figura post-metafísica de la voluntad y su inmanencia en la fenomenalización de la verdad? El "giro teológico", según lo entiende Janicaud, quedaría así radicalizado, superado, por un "giro espiritual" que devuelve a la fenomenología la posibilidad de comprender de manera inmanente lo que nos excede: el fenómeno saturado, es decir, su exceso de donación respecto a la conciencia.

Y cuarta conclusión: finalmente, nos permitimos lanzar esta vez una afirmación sin paliativos, no provisional sino definitiva hasta que no se demuestre rigurosamente lo contrario: el "giro teológico de la fenomenología francesa" en el caso concreto de Marion, ya sea simplemente un "giro teológico" o - según postulamos nosotros, más radicalmente- un "giro espiritual", no puede entenderse sin esa partícula enigmática, Janus del lenguaje, llave de la trascendencia ad nos, el "como".

\section{Bibliografía}

Bassas Vila, J., “Écriture phénoménologique et théologique", en C. Ciocan y M. Neamtu (eds.), Studia Phaenomenologica, 2009, volumen titulado Philosophical concepts and religious metaphors: new perspectives on phenomenology and theology.

De Libera, A., "Saint Augustin, penseur du soi. Discussions de l'interprétation de Jean-Luc Marion", Revue de métaphysique et de morale, 63 (2009/3).

Fink, E., "Operative Begriffe in Husserls Phänomenologie", Zeitschrift für philosophische Forschung, 11 (1957), pp. 321-337.

Grondin, J., "Au lieu de la métaphysique? Les méditations augustiniennes de Jean-Luc Marion", recensión del libro de J.-L. Marion, $A u$ lieu de soi. L'approche de Saint Augustin, consultado en línea (05-01-2009): http://www.laviedesidees.fr/Au-lieu-de-la-metaphysique.html

Marion, J.-L., Dieu sans l'être, Paris, Presses Universitaires de France, 1982; versión española: Dios sin el ser, traducción de D. Barreto González, J. Bassas Vila y C. E. Restrepo, revisión y postfacio de J. Bassas Vila, Castellón, Ellago, 2010.

Marion, J.-L., Étant donné. Essai d'une phénoménologie de la donation, Paris, Presses Universitaires de France, 1997; versión española: Siendo dado. Ensayo para una fenomenología de la donación, prólogo a la edición española de J.-L. Marion, traducción, presentación y notas de J. Bassas Vila, Madrid, Síntesis, 2008.

Marion, J.-L., Au lieu de soi. L'approche de Saint Augustin, Paris, Presses Universitaires de France, 2008.

Marion, J.-L., Le croire pour le voir, Paris, Parole et silence, 2010. 